\title{
Adiciones a los Gastropoda del mar peruano
}

\author{
Additions to Gastropoda from the Peruvian sea
}

\section{Carlos Paredes ${ }^{1}$, Franz Cardoso ${ }^{1}$, Paul Baltazar ${ }^{1}$, Katherine Altamirano ${ }^{1}$ y Patricia Carbajal $^{2}$}

\begin{abstract}
1 Laboratorio de Biología y Sistemática de Invertebrados Marinos, Facultad de Ciencias Biológicas, ICBAR, UNMSM, Apdo. 1100-58, Lima 11, Perú. Email: cparedesq@ unmsm.edu.pe

2 Unidad de Investigaciones en Biodiversidad, Instituto del Mar de Perú IMARPE. Esquina Gamarra y General Valle S/N Chucuito, Callao.
\end{abstract} Aceptado: $\quad 20 / 01 / 2011$ Publicado online: $23 / 06 / 201$

\begin{abstract}
Resumen
Se reportan por primera vez para el mar peruano seis especies de gasterópodos: Sthenorytis turbinus Dall, 1908, Crucibulum (Crucibulum) umbrella (Deshayes, 1830), Favartia (Murexiella) lappa (Broderip,1833), Cantharus (Cantharus) shaskyi Berry, 1959, Cantharus (Solenosteira) macrospira (Berry, 1957), Cancellaria (Bivetopsia) haemastoma Sowerby, 1832. Se informa sobre la distribución, el hábitat y comentarios relevantes acerca de cada una de las especies.
\end{abstract}

Palabras claves: Mollusca, Gastropoda, Caenogastropoda, nuevos registros, Perú.

\section{Abstract}

The following species of Gastropoda are recorded for the first time for Peruvian waters: Sthenorytis turbinus Dall, 1908, Crucibulum (Crucibulum) umbrella (Deshayes, 1830), Favartia (Murexiella) lappa (Broderip,1833), Cantharus (Cantharus) shaskyi Berry, 1959, Cantharus (Solenosteira) macrospira (Berry, 1957), Cancellaria (Bivetopsia) haemastoma Sowerby, 1832.

Keywords: Mollusks, Gastropoda, Caenogastropoda, new records, Peru.

\section{Introducción}

Continuando con el inventario y la determinación actualizada de los moluscos marinos (Paredes et al. 1999; Ramírez et al. 2003; Paredes \& Cardoso 2005; Paredes \& Cardoso 2007; Paredes et al. 2008, 2009, 2010), y teniendo como objetivos: elaborar catálogos ilustrados de las especies y organizar colecciones de referencia en el Museo de Historia Natural de la Universidad Nacional Mayor de San Marcos, y el Laboratorio de Biología y Sistemática de Invertebrados Marinos de la Facultad de Ciencias Biológicas; en esta oportunidad se da a conocer el hallazgo de seis especies de gasterópodos de la subclase Caenogastropoda, que son registradas por primera vez para el mar peruano.

\section{Material y métodos}

El material revisado de cinco especies procede en su mayoría de colectas realizadas en las aguas tropicales de la Provincia Peruana, frente a los Departamentos de Tumbes y Piura, al norte del Perú. Una de las especies procede del área de Pisco y fue colectada por Hans-Wilhelm Koepcke.

El material biológico fue fijado en el campo o en el laboratorio, utilizando formol al 7\% neutralizado con bórax. Para su conservación y estudio, las muestras fueron lavadas con agua corriente y luego conservadas en alcohol al 70\%. Para el ordenamiento supraespecifico se siguió a Skoglund (2002) y la determinación taxonómica se realizó utilizando la bibliografía especializada. Las fotografías se tomaron utilizando una cámara digital. El material está depositado el Laboratorio de Biología y Sistemática de Invertebrados Marinos de la Facultad de Ciencias Biológicas (LaBSIM) y el Museo de Historia Natural de la Universidad Nacional Mayor de San Marcos.

\section{Taxonomía}

\section{Clase Gastropoda}

Subclase CaEnogastropoda

Orden Neotaenioglossa

SUPERFAMILIA JANTHINOIDEA

FAMILIA EPITONIIDAE

SUBFAMILIA EPITONIINAE

Genero Sthenorytis Conrad, 1862

\section{Sthenorytis turbinus (Dall, 1908)}

Figura 1

Epitonium (Sthenorytis) turbinum Dall, 1908. Bull. Mus. Comp. Zool., Harvard, Vol. 43, № 6, pp. 205-487, pls. 1-22 (Oct.); Keen, 1971: 436, fig. 665. Sthenorytis turbinus, Skoglund, 2002: 61.

Vuelta corporal grande y globosa, espira cónica con cinco vueltas; abertura redondeada con labios brillantes; escultura axial desarrollada consiste en doce costillas lamelares, cóncavas y con los bordes afilados, los interespacios son lisos. El color general es blanco con una tonalidad grisácea en los interespacios de la vuelta corporal y las dos últimas vueltas de la espira; el interior de la abertura es blanco; el opérculo córneo tiene color marrón negruzco y presenta núcleo casi central y pocas líneas espirales. Longitud, 40,2 mm; diámetro 30,6 mm.

Material examinado: 2 lotes, 2 ejemplares.

Hábitat: Sublitoral, fondo rocoso arenoso, 110 - 550 metros de profundidad (Keen 1971).

Localidades: Piura (Máncora), 107 - 122 metros.

Fecha: 10/09/2008, 12/12/2009.

Distribución: Isla San Pedro Nolasco, Golfo de California hasta las Islas Galápagos, Ecuador (Keen 1971).

Observaciones: Nuevo registro para el mar peruano

\author{
Superfamilia CaLYPTRAEOIDEA \\ FAMILIA CALYPTRAEIDAE \\ Genero Crucibulum Schumacher, 1817 \\ Subgénero CRUCIBULUM, S. S.
}

Crucibulum (Crucibulum) umbrella (Deshayes, 1830)

Figura 2

Crucibulum umbrella Deshayes, 1830. Encyclopédie Méthodique. Histoire Naturelle de vers, vol. 2. Paris. Pp.1-144.

Crucibulum (Crucibulum) umbrella, Keen, 1971: 465, fig. 827; Skoglund, 2002:74; González-Villarreal, 2005:31, fig. 44. 


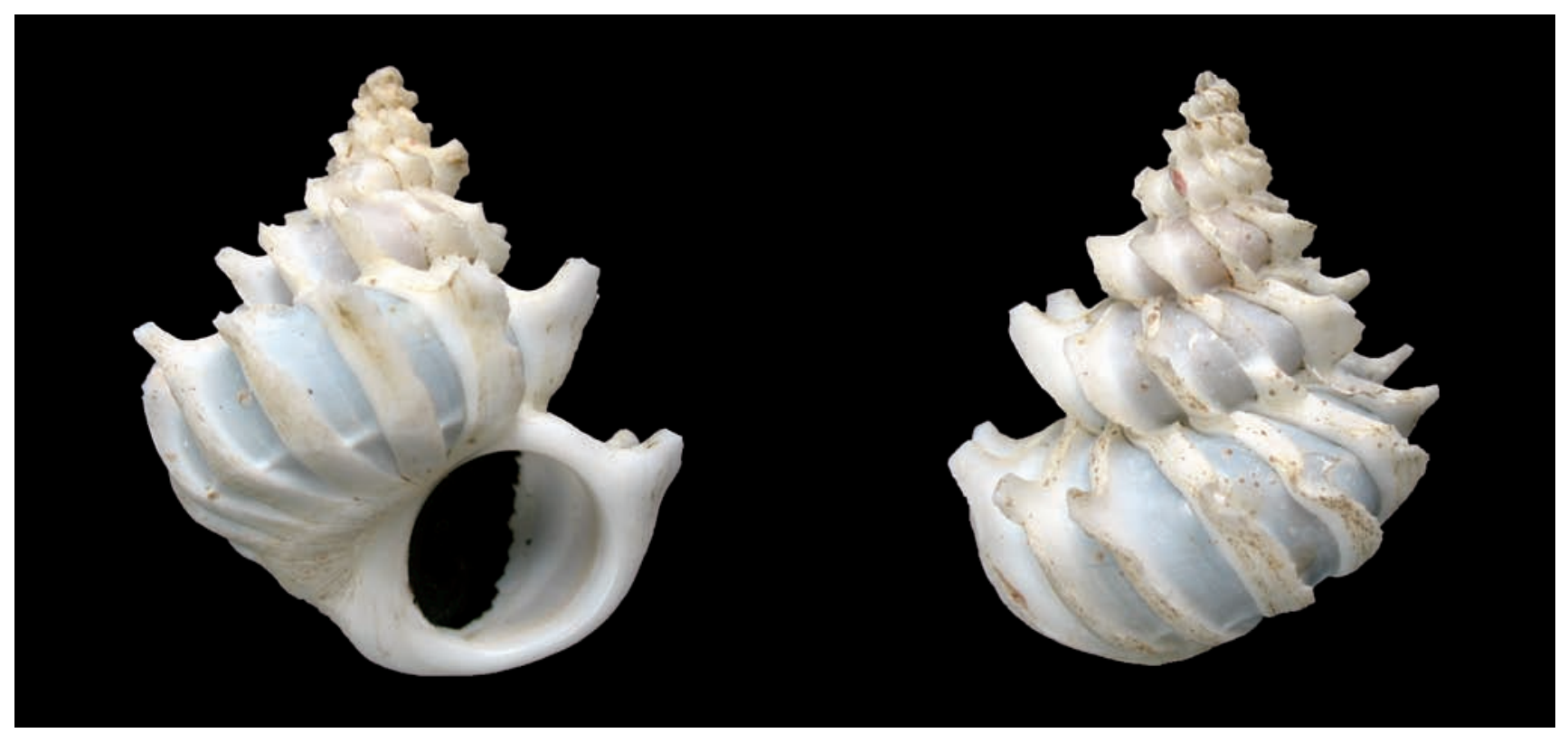

Figura 1. Sthenorytis turbinus.

Conchilla poco elevada con apex subcentral, margen crenulado por las proyecciones de las costillas; escultura con 26 a 34 costillas radiales, los interespacios tan anchos como las costillas; la copa interna está fijada bajo el apex y por uno de los lados. El color de las conchillas es blanco opaco por fuera y por dentro, la copa también es blanca. Diámetro 43,0 mm; altura 12,5 mm.

Material examinado: 1 lote, 2 conchillas varadas en la playa arenosa (Kp. 1491).

Hábitat: Intermareal rocoso (González-Villarreal 2005).

Localidades: Ica, Pisco (Laguna Grande).

Fecha: entre el 29 de diciembre de 1957 y el 2 de enero de 1958

Distribución: Golfo de California hasta Panamá (Keen 1971).

Observaciones: Las conchillas fueron colectadas por HansWilhelm Koepcke (1982), habiendo permanecido en el Museo de Historia Natural. Nuevo registro para el mar Peruano.

\author{
Suborden NeOgastropoda \\ SUPERFAMILIA MURICOIDEA \\ FAMILIA MURICIDAE \\ SubFAMILIA MURICOPSINAE \\ GÉnero FaVARTIA JousseAUMe, 1880 \\ Subgénero Murexiella Clench y Pérez Farfante, 1945
}

Favartia (Murexiella) lappa (Broderip, 1833)

Figura 3

Murex lappa Broderip, 1833. Proc. Zool. Soc. London: p. 177. Phyllonotus lappa, Dall, 1909: 219.

Murexiella lappa, Keen, 1971:519, fig. 990; Hendrickx y Toledano,

1994: 46; Mair et al., 2002: 21, lám. 14, fig. 4.

Murex lappa, Abbott, 1974: 175.

Favartia (Murexiella) lappa, Skoglund, 2002: 109.

Concha con espira pequeña que alcanza aproximadamente la cuarta parte de la longitud total; escultura presenta seis várices de las que se proyectan fuertes espinas cóncavas y acanaladas ventralmente; la abertura es oval con canal anterior desarrollado y

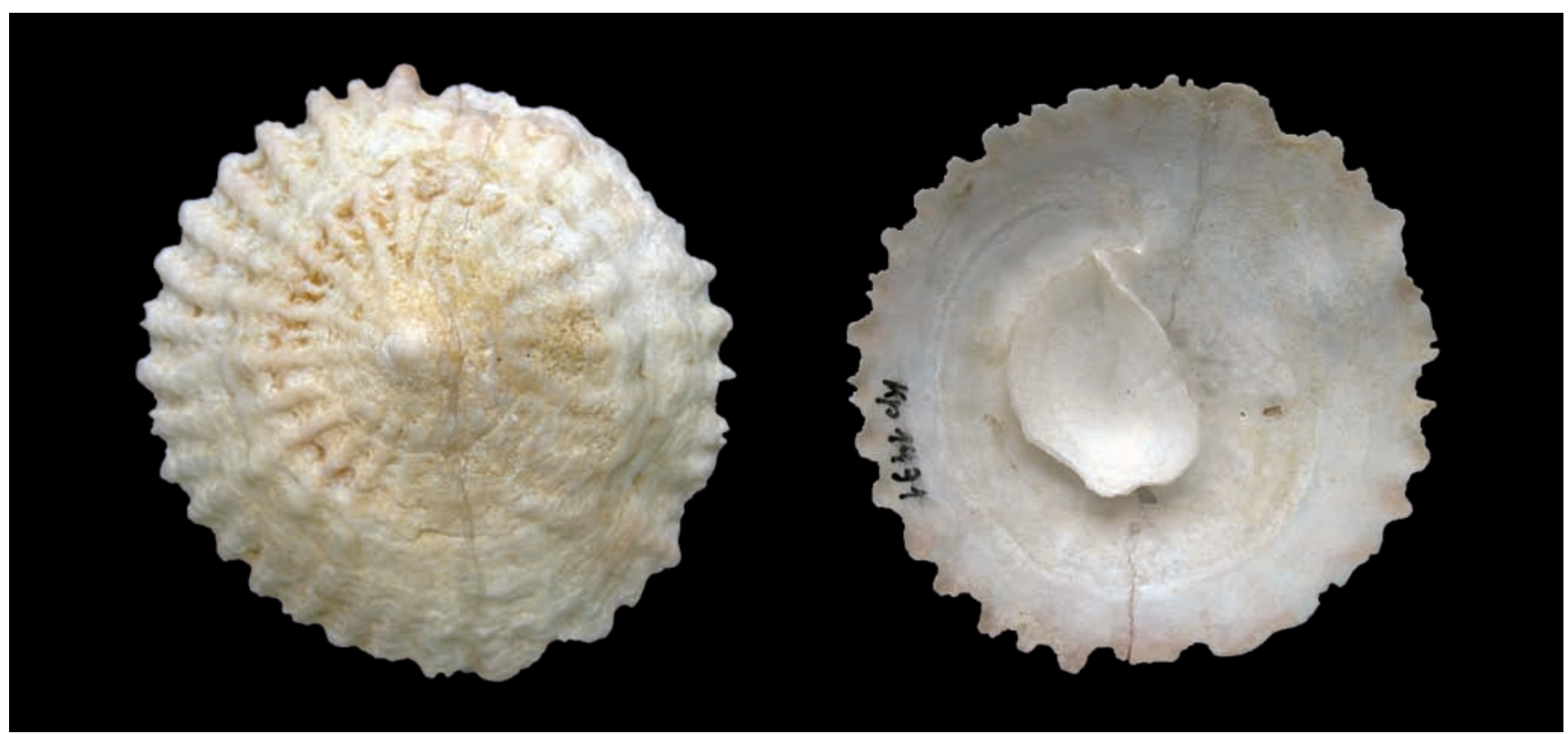

Figura 2. Crucibulum (Crucibulum) umbrella. 


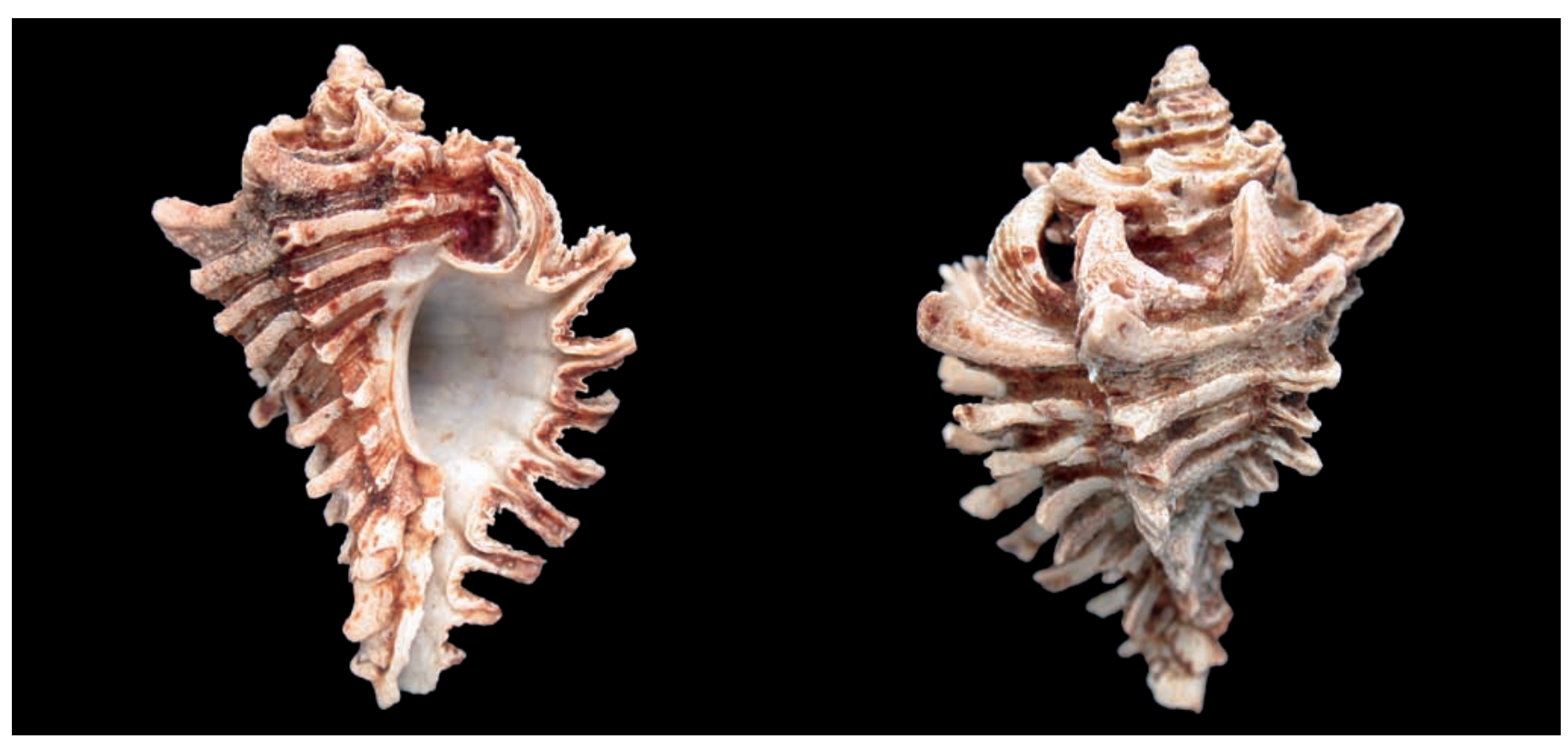

Figura 3. Favartia (Murexiella) lappa.

reflejado dorsalmente en su tercio distal, el labio columelar es liso y el externo crenulado por efecto de las espinas. Color externo pardo claro y el interior de la abertura blanquecino ligeramente violáceo presenta líneas geométricas que son producto de la complicada escultura externa. Longitud, $40,3 \mathrm{~mm}$.

Material examinado: 1 lote, 1 ejemplar.

Hábitat: Intermareal inferior y sublitoral hasta 20 metros de profundidad (Keen, 1971).

Localidades: Piura (Bahía de Paita), fondo areno rocoso.

Fecha: Año 2004.

Distribución: Guaymas, México hasta Panamá (Keen 1971), Islas Galápagos, Ecuador (Finet 1985).

Observaciones: El material fue colectado y guardado por personal del Laboratorio Costero de IMARPE. Nuevo registro para el mar peruano.
FAMILIA BUCCINIDAE

Subfamilia PisaniINAE

GÉnero Cantharus Röding, 1798

SugÉnERo CANTHARUS, S. S.

\section{Cantharus (Cantharus) shaskyi Berry, 1959}

\section{Figura 4}

Cantharus (Cantharus) shaskyi Berry, 1959. Notices of new eastern Pacific Mollusca III. Leaflets in Malacol., vol. 1, № 18, pp.108-13; Keen, 1971: 560, fig. 1106; Abbott, 1974:220; Hendrickx y Toledano, 1994: 48; Skoglund, 2002: 123.

Conchilla con la vuelta corporal globosa y la espira de forma similar tiene una altura aproximada equivalente a un tercio de la longitud total, la sutura es bien definida; abertura oval y canal sifonal corto, el fasciolo sifonal está desarrollado; el labio columelar es cóncavo y el externo presenta estrías; escultura consiste en 12 costillas axiales bajas que están cruzadas por densas estrías espirales algo irregulares, algunas de las cuales son más prominentes sobre las costillas, presentando un patrón espiral blanco característico.

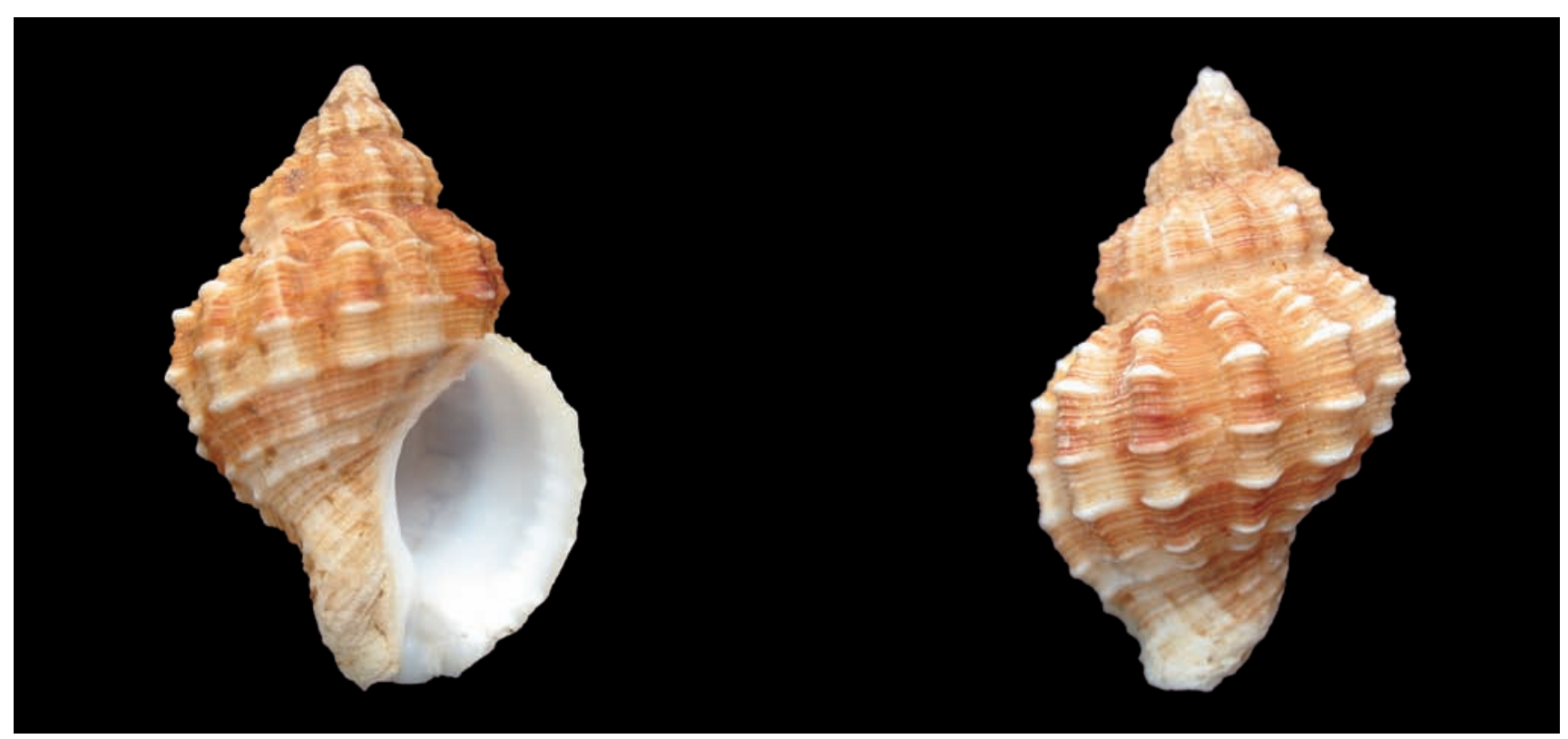

Figura 4. Cantharus (Cantharus) shaskyi. 


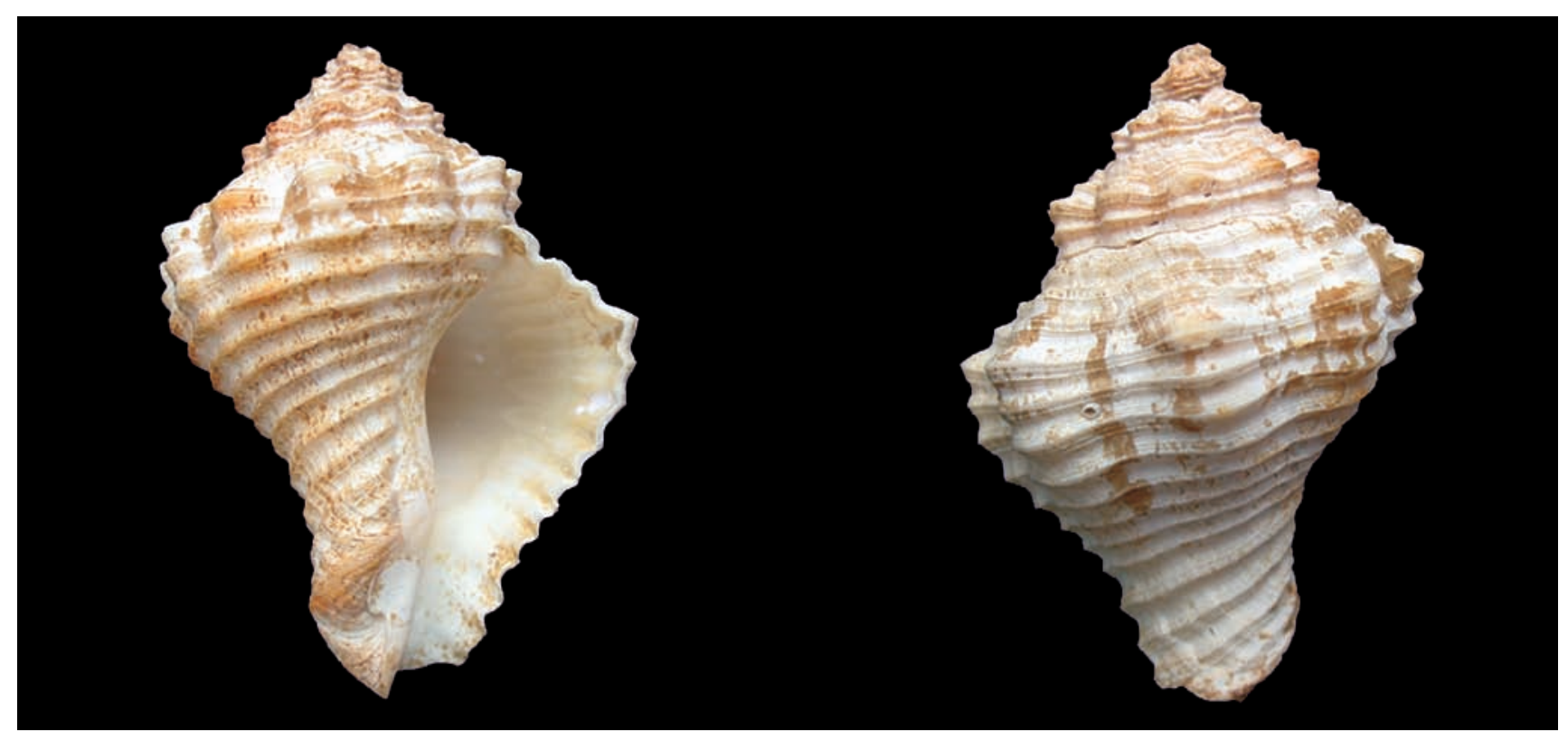

Figura 5. Cantharus (Solenosteira) macrospira.

Subgénero Solenosteira Dall, 1890

El color general es pardo claro con líneas o manchas oscuras y claras, interior de la abertura es blanco brillante en los labios y el canal sifonal. Longitud 35,7 mm; ancho 22,7 mm.

Material examinado: 1 lote, 3 ejemplares.

Hábitat: Intermareal rocoso (Hendrickx y Toledano 1994).

Localidades: Piura (Máncora), fondo arenoso rocoso, 120 -122 metros.

Fecha: 10/09/2008.

Distribución: Frente a Bahía San Carlos, Sonora, México (Skoglund 2002); Bahía Asunción, Baja California Sur, México (Luke 1995).

Observaciones: Nuevo registro para el mar peruano.
Cantharus (Solenosteira) macrospira (Berry, 1957)

Figura 5

Solenosteira macrospira Berry, 1957. Notices of new eastern Pacific Mollusca. I. Leaflets in Malacol., vol. 1, № 14, pp. 75-82; Keen, 1971: fig.1121; Hendrickx y Toledano, 1994: 49-50.

Cantharus (Solenosteira) macrospira, Skoglund, 2002:124.

Según Keen (1971), la conchilla es muy semejante a Solenosteira pallida, diferenciándose por ser ligeramente más corta para su ancho, vuelta corporal desarrollada y espira corta con tres a cinco vueltas; el canal sifonal es corto, amplio, y reflejado hacia la izquierda en su parte terminal; el fasciolo columelar está bien desarrollado, el labio interno es liso y el externo crenulado; la vuelta corporal presenta nueve costillas axiales reducidas que se inician cerca de la base de la misma y terminan en los hombros;

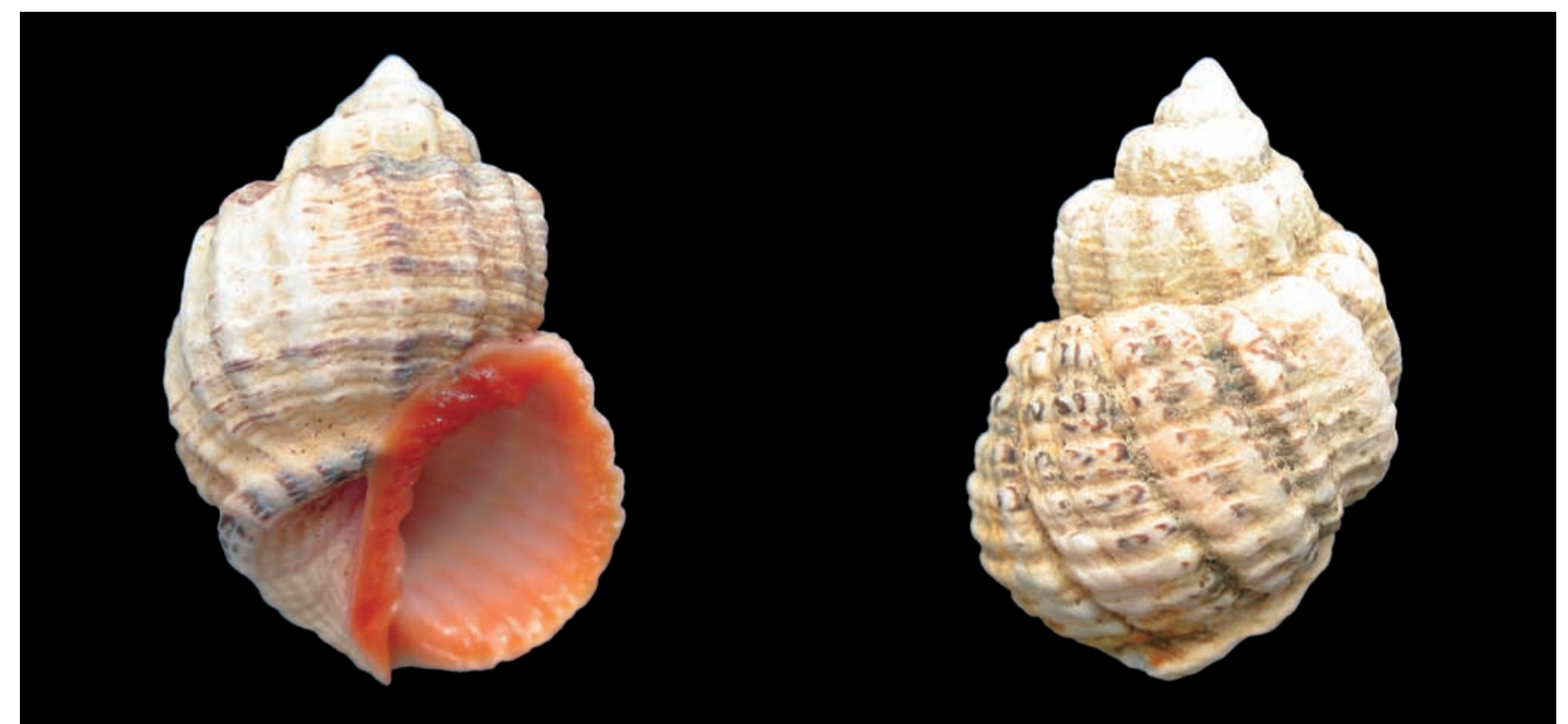

Figura 6. Cancellaria ( Bivetopsia) haemastoma. 
esta escultura se repite en las vueltas de la espira, y toda la conchilla presenta marcadas costillas espirales en toda la superficie. La coloración general es pardo clara con manchas blancas y el periostraco tiene color pardo oscuro, el interior de la abertura es blanco amarillento. Longitud $38,5 \mathrm{~mm}$; diámetro $28,0 \mathrm{~mm}$.

Material examinado: 1 lote, 1 ejemplar.

Hábitat: Sublitoral areno fangoso, hasta 33 metros ( Hendrickx y Toledano, 1994).

Localidades: Tumbes (Puerto Pizarro), varado en la playa.

Fecha: 09/08/1992.

Distribución: Extremo norte del Golfo de California (Keen 1971) hasta el sur de Bahía Ballena, Costa Rica (Cruz 1996).

Observaciones: Nuevo registro para el mar peruano.

\author{
Superfamilia CANCELlarioidea \\ FAMILIA CANCELLARIIDAE \\ GÉNERo CANCELLARIA LAMARCK, 1799 \\ Subgénero Bivetiella Wenz, 1943 \\ Subgénero BIVETOPSIA JousSEAUME, 1887
}

\section{Cancellaria ( Bivetopsia) haemastoma Sowerby, 1832}

\section{Figura 6}

Cancellaria (Bivetopsia) haemastoma Sowerby, 1832. Proc. Zool. Soc. London: 54; Keen, 1971: 651, fig.1464; Skoglund, 2002: 157.

Conchilla con amplia vuelta corporal y hombros prominentes, inclusive en la corta espira, abertura pequeña con un corto canal sifonal, labio interno con estrías basales y tres pliegues desarrollados en la parte distal, labio externo crenulado; escultura presenta costillas axiales no muy prominentes ampliamente separadas, y también suaves costillas espirales, el ombligo es estrecho y profundo y el fasciolo sifonal bien desarrollado alcanza casi la longitud de la abertura. El color general es pardo claro con manchas o líneas oscuras; labios con un color rojizo anaranjado. Longitud, 30, $8 \mathrm{~mm}$; diámetro, 21, $4 \mathrm{~mm}$.

Material examinado: 2 lotes, 12 ejemplares.

Hábitat: Sublitoral, fondo arenoso y conchuela.,

Localidades: Piura (Sechura, Vichayo, Parachique), 10 - 20 $m$ de profundidad.

Fecha: 18/12/2007.

Distribución: Islas Galápagos, Ecuador (Keen 1971).

Observaciones: Según Keen (1971), ha sido considerada una subespecie de Cancellaria (B.) chrysostoma, debido a la similitud morfológica. Actualmente es una especie válida (Skoglund 2002). Nuevo registro para el mar peruano.

\section{Agradecimientos}

Los autores agradecen al Consejo Superior de Investigaciones de la Universidad Nacional Mayor de San Marcos por el apoyo económico a nuestros proyectos de investigación sobre diversidad de moluscos marinos. Expresamos nuestro reconocimiento al doctor Dimitri Gutierrez, Jefe del Laboratorio de Bentos Marino del Instituto del Mar del Perú por habernos proporcionado un ejemplar de Sthenorytis turbinus y el matrial de Cantharus shaskyi. Igualmente agradecemos al biologo Isaias Gonzales, Jefe del Laboratorio Costero del Instituto del Mar del Perú en Paita.

\section{Literatura citada}

Abbott R.T. 1974. American Seashells: The marine Mollusca of the Atlantic and Pacific coast of North America. Van Nostrand Reinholt Co.. New York. 663 pp.

Alamo V. \& V. Valdivieso. 1987. Lista sistemática de moluscos marinos del Perú. Bol. Inst. Mar. Perú. Vol. extraordinario: $1-205$.

Cruz R.A. 1996. Annotated checklist of marine mollusks collected during the R.V. Victor Hensen Costa Rica Expedition $1993-$ 1994. Revista de Biología Tropical 44, Suppl. 3: 59-68.

Dall W.H. 1908. Reports on the dredging off the west coast of the Central America to the Galapagos, to the west coast of Mexico, and in the Gulf of California, in charge of Alexander Agassiz, carried onby the U. S. Fish Comission steamer “Albatros", during 1891, Lieut Commander Z. I. Tanner, U. S. N., commanding. The Mollusca and Brachiopoda. Bull. Mus. Comp. Zool. , Harvard, vol. 43, Nº 6, pp. $205-$ 487, pls. 1-22 (Oct.)

Dall W.H. 1909. Report on a collection of shells from Peru, with a summary of the littoral marine mollusca of the Peruvian Zoological province. Proc. U.S.N. Mus. 37 (1704): 147294.

Finet Y. 1985. Preliminary faunal list of the marine molusks of the Galápagos Islands. Institut Royal des Sciences Naturelles de Belgique. Documents de Travail 20: 50 pp.

González-Villarreal L.M. 2005. Guía ilustrada de los gasterópodos marinos de la Bahía de Tenacatita, Jalisco, México. Scientia- CUCBA 7(1):1-84.

Hendrickx M. \& A. Toledano.1994. Catálogo de Moluscos Pelecypodos, Gasterópodos y Poliplacóforos. Catálogo de Referencia, Estación Mazatlán, ICML; UNAM. Inst. Cien. del Mar y Limnología UNAM.. 71 pp.

Hernandez M.A. 1979. Range extensions of mollusks species found on the tropical coast of El Salvador. The Veliger 22(2): 204-205.

Keen A.M. 1971. Sea shells of tropical west America. 2th ed. Stanford Univ. Press, California. 1064 pp.

Koepcke H. W. und M. Koepcke. 1982. Erläuterung der Beobachtungs- und Samler- nummern zu den in den Jahren 1949 bis 1974 in Peru durchgeführten biologischen Studien. Nach den Geländenotizen zusammengestellt von H. W. Koepcke. Hamburg. 136 pp.

Luque S.R. 1995. Catalog of the Benthic Invertebrate Collections of the Scripps Institution of Oceanography Reference Series $\mathrm{N}^{\circ}$ 95-24, $176 \mathrm{pp}$.

Mair J., E. Mora \& M. Cruz. 2002. Manual de campo de los Invertebrados Bentónicos Marinos: Moluscos, Crustaceos y Equinodermos de la Zona Litoral Ecuatoriana. (Eds.) Editorial de la Universidad de Guayaquil. 108 pp.

Mogollón V., N. Chirichigno, L. Clemente \& G. Peña. 1999. Gasterópodos (Mollusca) de Isla Los Chimus y alrededores (Santa, Ancash, Perú). Wiñay Yachay 3(2):35-75.

Mulliner M. 1996. Dredging around Isla Danzante, Gulf of California, México or what you find at the end of a line. The Festivus 28(6): 62-70, figs. 1-17.

Paredes C. y F. Cardoso. 1998. Nuevos registros de gasterópodos para el litoral peruano. Rev. peru. biol. 5(2): 118-122.

Paredes C., P. Huamán, F. Cardoso, R. Vivar \& V. Vera. 1999. Estado actual del conocimiento de los moluscos acuáticos en el Perú. Rev. peru. biol. 6(1): 5-47.

Paredes C. \& F. Cardoso. 2007. La Familia Calyptraeidae en el Perú. Rev. peru. biol. 13 (3): 177-184. 
Paredes C. \& F. Cardoso. 2009. Nuevos registros de gasterópodos para el mar tropical del Perú. Rev. peru. biol. 15(2): 073078.

Paredes, C., F. Cardoso, K. Altamirano, P. Baltazarr \& L. Romero. 2010. La familia Conidae en el mar peruano. Rev. peru. biol. 17(1): 065-073.
Ramírez R., C. Paredes y J. Arenas. 2003. Moluscos del Perú. Rev. Biol. Trop. (Suppl. 3): 225-284.

Skoglund C. 2002. Panamic Province Molluscan literatura. Additions and changes From 1971 through 2001. III Gastropoda. The Festivus 33: 1-286. 